Nortes Martínez-Artero, R. \& Nortes Checa, A. (2020). Actitud hacia las matemáticas en el Grado de Maestro de Primaria. Revista Electrónica Interuniversitaria de Formación del Profesorado, 23(2), 225-239.

DOI: https://doi.org/10.6018/reifop.348061

\title{
Actitud hacia las matemáticas en el Grado de Maestro de Primaria
}

\author{
Rosa Nortes Martínez-Artero, Andrés Nortes Checha \\ Universidad de Murcia
}

\section{Resumen}

La actitud hacia las matemáticas de los futuros maestros puede condicionar la enseñanza de esta materia en los primeros niveles educativos. Para conocer cual es la actitud de los alumnos del Grado de Maestro de Primaria se ha consultado a lo largo de seis cursos académicos a 1150 alumnos de la facultad de Educación de la universidad de Murcia de $2 .^{\circ}, 3 .^{\circ}$ y $4 .^{\circ}$. Se les ha aplicado el cuestionario de Auzmendi (1992) que consta de 25 ítems en una escala de Likert de 1 a 5 . Los resultados indican que uno de cada tres alumnos tiene una actitud negativa (inferior a 3 ), y que los alumnos de $4 .^{\circ}$ tienen una actitud más alta. El ítem de mejor puntuación es uno del factor confianza y el de peor uno del factor agrado.

\section{Palabras clave}

Actitud; matemáticas; maestro; dominio afectivo.

\section{Future teachers' attitudes towards Mathematics}

\begin{abstract}
Future teachers' attitudes towards mathematics can influence the teaching of this subject-matter in the earlier years. In order to know their attitudes, 1,150 Primary Education students (2nd, 3rd and 4th years) of the Faculty of Education at the University of Murcia were surveyed during 6 academic years. They were given the Auzmendi (1992) test which is composed of 25 items on a 1-5 Likert scale.
\end{abstract}

Contacto:

Rosa Nortes Martínez-Artero, mrosa.nortes@um.es, Facultad de Educación. Campus de Espinardo. 30100. Murcia. 
The results show that 1 in 3 students has a negative attitude (lower than 3 ). They also show that men have a more favourable attitude than women and that 4th-year students have the highest attitude. The item with the highest score refers to the self-confidence factor whereas the one with the lowest score relates to the liking factor.

\section{Key words}

Attitude; mathematics; teacher; affective domain .

\section{Introducción}

En la enseñanza y aprendizaje de las matemáticas el dominio afectivo es tan importante como el cognitivo y las actitudes hacia las matemáticas son un elemento motor en la resolución de problemas, ya que una actitud positiva o negativa puede determinar el resultado al que finalmente se llega y si se es capaz o no de encontrar una solución (Estrada y Díez-Palomar, 2011).

Las actitudes, son "predisposiciones, favorables o desfavorables, que determinan las intenciones personales de los sujetos y son capaces de influir sus comportamientos o acciones frente al objeto, sujeto o situación" (Martínez, 2008, p. 244). Suelen ser relativamente estables, actúan como motivadoras de la conducta, pueden expresarse mediante lenguaje, no siempre tienen relación directa con la conducta emitida por el sujeto, no son observables de forma directa y conforman sus experiencias y la reflexión sobre ellas. Y Alemany y Lara (2010) perciben los siguientes elementos en común en relación con las actitudes: predisposición hacia la acción, la intensidad con que se muestra, carga afectiva favorable o desfavorable, se tienen hacia aquello de lo que se tiene experiencia, son inevitables, son adquiridas, responden a la necesidad que tiene la persona de estructurar el entorno y se diferencia en ellas tres factores básicos (cognitivo, afectivo y conductual). Las actitudes hacia las matemáticas pueden servir de partida para saber qué se puede hacer en el presente y sobretodo en el futuro.

\section{Antecedentes}

Uno de los investigadores que más ha trabajado en los últimos treinta años el dominio afectivo relacionado con las matemáticas indica que "en las actitudes hacia las matemáticas predomina el componente afectivo y se manifiestan en el interés, la satisfacción o la curiosidad o bien en el rechazo, la negación, la frustración o la evitación de la tarea matemática" (Blanco, 2015, p. 14).

Al referirse a las actitudes hacia las Matemáticas (Martínez, 2008) especifica que tienen que ver con "la valoración, el aprecio, la satisfacción, la curiosidad y el interés tanto por la disciplina como por su aprendizaje, acentuando más el componente afectivo que el cognitivo" (p. 250).

Desde que Auzmendi (1992) construyó en España una escala de actitudes hacia la estadística y las matemáticas, han sido muchos investigadores los que la han aplicado o construido su propio cuestionario, la mayor parte de ellos a partir de los trabajos de Fennema y Sherman (1976). Han tratado de ver en sus investigaciones la influencia que tienen a distintos niveles, tanto de primaria como de secundaria como de universidad, pero hay un colectivo de estudiantes cuyo estudio es primordial, se trata de los futuros maestros que van a ser los encargados de la enseñanza de las matemáticas a niveles básicos y en donde una actitud positiva hacia las matemáticas hará que los alumnos de Primaria se 
entusiasmen con esta materia tan necesaria y útil. En el Real Decreto 126/2014 del currículo básico de Educación Primaria en el primer bloque de "Procesos, métodos y actitudes" se especifica como contenido "confianza en las propias capacidades para desarrollar actitudes adecuadas y afrontar las dificultades propias del trabajo científico" (p. 19388).

Auzmendi (1992) a una muestra de 1221 estudiantes de primero, segundo y tercero de BUP (Bachillerato Unificado Polivalente) y COU (Curso de Orientación Universitario) aplicó un cuestionario que era una adaptación de los 25 ítems de que consta la Escala de Actitudes hacia la Estadística que previamente había confeccionado. La utilizó para analizar las actitudes mediante 9 ítems de ansiedad, 4 de agrado, 6 de utilidad, 3 de motivación y 3 de confianza y la fiabilidad fue medida con el índice alfa de Cronbach que resultó 0.9283 .

Fernández y Aguirre (2010) aplican el cuestionario de Auzmendi a una muestra de 146 estudiantes de primer curso del Grado de Maestro de Educación Primaria de la universidad de Castilla-La Mancha, donde el $68 \%$ son mujeres y el 32\% hombres de entre 18 y 20 años. Obtienen una actitud media de 3.014, ansiedad de 2.856, agrado de 2.90, utilidad de 2.952, motivación de 3.17 y confianza de 3.62. La correlación entre motivación y utilidad es de 0.5 y entre confianza y agrado de 0.36 , y no hay diferencias significativas por género, ni por provenir de bachillerato con o sin Matemáticas.

León-Mantero, Maz-Machado y Jiménez-Fanjul (2015) a una muestra de 183 estudiantes del Grado de Maestro de Primaria, 52 de $1 .^{\circ}$ y 131 de $3 .^{\circ}$, matriculados el curso 2013/14, aplicaron la escala de actitud hacia las matemáticas de Auzmendi (1992), obteniendo un alfa de Cronbach de 0,919 y en los cinco factores: utilidad (0.806), ansiedad (0.911), agrado (0.805), motivación (0.713) y confianza (0.108).

Maz-Machado, León-Mantero, Casas y Gática (2014) a una muestra de 51 estudiantes de 1. ${ }^{\circ}$ de Ingeniería Informática de la universidad de Córdoba les aplicaron la escala de Auzmendi (1992). De los ítems de utilidad, ansiedad, agrado y motivación analizados el más valorado fue "Considero las matemáticas como una asignatura muy necesaria en mis estudios" con 4,18 y la menos valorada con 2.35 "Me divierte el hablar con otros de matemáticas" y en donde un $34.96 \%$ dan una valoración neutra sobre la utilidad de las matemáticas, si bien la media es 3.38, mientras que para agrado fue de 2.66 .

León-Mantero, Pedrosa-Jesús, Maz-Machado y Casas-Rosal (2018) a una muestra de 408 estudiantes del Grado de Maestros de Primaria aplicaron la Escala de Auzmendi para obtener, entre otras, una reordenación de los ítems que conforman la escala, eliminando tres de ellos y pasando de los seis de utilidad a tres, de los nueve de ansiedad a seis, de los cuatro de agrado a cinco, de los tres de confianza a cinco y manteniendo los tres de motivación, obteniendo como la correlación más alta agrado-confianza de .747. También Fernández Cézar, Solano, Rizzo, Gomezescobar, Iglesias y Espinosa (2016) revisaron la Escala de Auzmendi y llegaron a la conclusión de reducir de 25 a 7 ítems para la dimensión de ansiedad, eliminando el resto y recomendando "estos siete ítems como un buen constructo para medir la ansiedad en maestros en ejercicio y estudiantes para maestro de educación infantil y primaria" (p. 235) eliminando así mismo dos de los inicialmente establecidos en ansiedad por Auzmendi, pasando, pues, de una escala de Actitud a una escala de Ansiedad. Posteriormente Gómezescobar y Fernández Cézar (2018) en una muestra a 53 docentes en activo diferenciados por sexo, años de ejercicio, etapa educativa, categoría profesoral y comunidad matemática en red aplican la escala de Auzmendi (1992) encontrando diferencias significativas en agrado de los maestros interinos respecto de los funcionarios y en agrado y confianza en la comparación atendiendo a presencia o no a la comunidad matemática, no existiendo diferencias significativas en cuanto a sexo o a etapa educativa. 
Casas, León-Mantero, Maz-Machado, Jiménez-Fanjul y Madrid (2016) aplicaron la escala de actitudes de Auzmendi a una muestra de 277 estudiantes de $10^{\circ}$ del Grado de Maestro de Primaria de Córdoba, de los que 112 son hombres y 165 mujeres, de edades comprendidas entre 18 y 48 años. Obtienen un índice de fiabilidad de Cronbach de 0.887 , fuertes correlaciones entre utilidad-confianza (0.797), utilidad-agrado (0.787), agrado-confianza (0.591) y utilidad-motivación (0.518) y correlaciones débiles entre los factores agradomotivación (0.261) y motivación-confianza (0.347).

Flores y Auzmendi (2015), a partir de una muestra de 182 estudiantes universitarios de especialidades en educación de Nicaragua de edades entre 16 y 24 años, se centran en analizar, validar y confirmar las propiedades psicométricas de la escala de Actitud hacia las Matemáticas de Auzmendi (1992). El índice de homogeneidad varía entre 0.32 y 0.77 y el índice de fiabilidad de Cronbach es de 0.914, obteniendo una varianza explicada del 63.234\%, y por factores ansiedad del $33.198 \%$, agrado del $10.236 \%$, utilidad del $8.513 \%$, motivación del $6.430 \%$ y confianza del $4.857 \%$.

\section{Objetivo}

El objetivo del presente estudio es conocer la actitud de los alumnos del Grado de Maestro de Primaria (GMP) a lo largo de seis cursos académicos al aplicarles el cuestionario de Auzmendi (1992) y comparar con otros estudios anteriores. Se pretende analizar a lo largo de los años la actitud que presentan hacia las matemáticas los futuros maestros, la ansiedad, el agrado, la utilidad, la motivación y la confianza con que se enfrentan a las Matemáticas en los cursos del GMP.

\section{Metodología}

\section{Participantes}

Son 1150 estudiantes del GMP matriculados en los curso 2011/12, 2012/13, 2013/14, 2014/15, 2015/16 y 2016/17 en la universidad de Murcia, correspondientes a $2 .^{\circ}, 3 .^{\circ}$ y $4 .^{\circ}$, de edades comprendidas entre 17 y 53 años y edad media de 21.8 años. La muestra es incidental. El reparto viene en tabla 1.

Tabla 1.

Participantes por curso académico, curso del grado y sexo

\begin{tabular}{cccccccccc}
\hline & $\mathbf{1 1 / 1 2}$ & $\mathbf{1 2 / 1 3}$ & $\mathbf{1 3 / 1 4}$ & $\mathbf{1 4 / 1 5}$ & $\mathbf{1 5 / 1 6}$ & $\mathbf{1 6 / 1 7}$ & TOT & HOM & MUJ \\
\hline $2 .^{\circ}$ & 69 & 189 & 71 & 60 & 60 & 59 & 508 & 126 & 382 \\
\hline $3 \cdot^{\circ}$ & 78 & 79 & 76 & 35 & 83 & 75 & 426 & 101 & 325 \\
\hline $4 \cdot^{\circ}$ & --- & 41 & 50 & 47 & 38 & 40 & 216 & 74 & 142 \\
\hline TOT & 147 & 309 & 197 & 142 & 181 & 174 & 1150 & 301 & 849 \\
\hline
\end{tabular}

El que en la tabla no figure ningún alumno de $4 .{ }^{\circ}$ en el curso $11 / 12$ es debido a que el GMP no estaba totalmente implantado 


\section{Instrumento}

Cuestionario de Actitud hacia las Matemáticas de Auzmendi (1992), que consta de 25 ítems. Unos redactados de forma positiva y otros de forma negativa (A2, A5, A7, A10, A12, A15, A16, A15, A22 y A25) en una escala tipo Likert de 1 a 5, (1=Totalmente en desacuerdo, 2=En desacuerdo, $3=\mathrm{Ni}$ de acuerdo, ni en desacuerdo, $4=$ De acuerdo y $5=$ Totalmente de acuerdo) agrupados en cinco factores: ansiedad ( $A 2, A 3, A 7, A 8, A 12, A 13, A 17, A 18, A 22)$, agrado ( $A 4$, A9, A14, A24), utilidad ( $A 1, A 6, A 15, A 16, A 19, A 21)$, motivación ( $A 5, A 10, A 25)$ y confianza (A11, A20, A23). En los ítems negativos se ha invertido su puntuación para su análisis (1 pasa a 5,2 pasa a $4 . .$. ), teniendo cada factor asignado un número de ítems por lo que se suman las puntuaciones en cada uno de ellos y se divide por el número de ítems para poder efectuar las medias que varían de 1 a 5 . Así en Ansiedad la puntuación menor es 9 y la mayor es 45 , y las medias correspondientes son 1 y 5 , respectivamente, mientras que en confianza la puntuación varía de 3 a 15 y las medias de 1 a 5 . Los ítems del Cuestionario se presentan en el Anexo.

\section{Procedimiento}

Este estudio se inició en el curso 2011/12 con la aplicación de la escala de actitud hacia las Matemáticas de Auzmendi (1992), que como indican Palacios et al. (2014), "es la escala de actitudes hacia las matemáticas más citada de las realizadas en lengua castellana” (p. 70). A partir de ahí cada curso académico en los primeros días de clase, los autores del presente estudio, aplican esta prueba junto a otras de conocimientos matemáticos elementales a alumnos de $2 .^{\circ}, 3 .^{\circ}$ y $4 .^{\circ}$ del GMP, cursos en los que los alumnos tienen asignaturas de Matemáticas y su didáctica (I y II) y Taller de Matemáticas, respectivamente. El curso 11/12 participó un grupo de $2 .^{\circ}$ y otro de $3 .^{\circ}$, el curso $12 / 13$ tres grupos de $2 .^{\circ}$, dos de $3 .^{\circ}$ y uno de $4 .^{\circ}$ y los cursos $14 / 15,15 / 16$ y $16 / 17$, uno de $2 .^{\circ}$, dos de $3 .^{\circ}$ y uno de $4 .^{\circ}$. Se utiliza en la obtención de resultados y su análisis el paquete estadístico Systat v13.

\section{Resultados}

\section{Resultados globales}

Se ha obtenido la tabla 2 con los resultados globales de los 1150 alumnos considerando la variable Actitud hacia las matemáticas (AU) y los factores Ansiedad (AN), Agrado (AG), Utilidad (UT), Motivación (MO) y Confianza (CO).

Tabla 2.

Resultados globales de actitud y factores que lo componen

\begin{tabular}{lccccccc}
\hline & EDAD & AU & AN & AG & UT & MO & CO \\
\hline $\mathrm{N}$ & 1121 & 1150 & 1150 & 1150 & 1150 & 1150 & 1150 \\
\hline Min. & 17 & 1.56 & 1 & 1 & 1.33 & 1 & 1.33 \\
\hline Máx. & 53 & 4.84 & 5 & 5 & 5 & 5 & 5 \\
\hline Media & 21.75 & 3.21 & 2.97 & 2.62 & 3.39 & 3.83 & 3.94 \\
\hline DT & 4.69 & 0.549 & 0.80 & 0.79 & 0.64 & 0.67 & 0.62 \\
\hline
\end{tabular}




\begin{tabular}{cccccccc}
\hline & EDAD & AU & AN & AG & UT & MO & CO \\
\hline Mediana & 20 & 3.200 & 3 & 2,75 & 3.33 & 4 & 4 \\
\hline
\end{tabular}

- En el intervalo (Media-DT, Media+DT) $=(2.66,3.75)$, se encuentran 788 alumnos, que representan el $68.52 \%$ del total.

- En el intervalo inferior $(1$, Media-DT $)=(1,2.66)$ se encuentran 179 alumnos, el $15.57 \%$ del total.

- En el intervalo superior (Media+DT, 5) $=(3.76,5)$ se encuentran 183 alumnos, el $15.91 \%$ del total.

- Hay 394 alumnos con calificación inferior a 3 (actitud negativa). El 34.26\%.

- La mediana es 3.20, el cuartil inferior es 2.84 y el cuartil superior es 3.60.

- La moda es doble 2.96 y 3.00, con 42 alumnos.

En la tabla 3 se presentan los resultados (Media y DT) por ítems.

Tabla 3.

Resultados por ítem de actitud

\begin{tabular}{lccccccccc}
\hline & A1 & A2 & A3 & A4 & A5 & A6 & A7 & A8 & A9 \\
\hline Media & 4.15 & 2.95 & 2.86 & 2.50 & 3.88 & 3.67 & 2.53 & 2.84 & 2.25 \\
\hline DT & 0.87 & 1.12 & 1.11 & 1.03 & 0.96 & 0.98 & 1.30 & 1.06 & 1.03 \\
\hline & A10 & A11 & A12 & A13 & A14 & A15 & A16 & A17 & A18 \\
\hline Media & 4.18 & 3.73 & 3.22 & 2.77 & 2.66 & 3.315 & 3.15 & 3.20 & 2.86 \\
\hline DT & 0.88 & 0.90 & 1.02 & 1.00 & 1.00 & 1.04 & 1.06 & 1.07 & 1.03 \\
\hline & A19 & A20 & A21 & A22 & A23 & A24 & A25 & & \\
\hline Media & 2.71 & 4.33 & 3.38 & 3.14 & 3.75 & 2.78 & 3.43 & & \\
\hline DT & 1.06 & 0.96 & 0.98 & 1.10 & 0.91 & 1.07 & 0.95 & & \\
\hline
\end{tabular}

A partir de la tabla y con los datos iniciales, previos cálculos sencillos, se obtiene que:

- El ítem mejor valorado es el A20 y el peor valorado A9.

- El ítem menos disperso en las respuestas es A1 y el más disperso A7.

- Más del 80\% de los alumnos señalan con 405 los ítems A1 y A20.

- Menos del $15 \%$ señalan con 4 o 5 los ítems A9 y A4.

\section{2. Índice de fiabilidad por factor y curso académico}

En la tabla 4 se presenta el índice de fiabilidad alfa de Cronbach por curso académico y factor. 
Tabla 4.

Fiabilidad de los ítems por factor y total

\begin{tabular}{llllllll}
\hline & $11 / 12$ & $12 / 13$ & $13 / 14$ & $14 / 15$ & $15 / 16$ & $16 / 17$ & TOT \\
\hline AU & 0.91 & 0.92 & 0.89 & 0.88 & 0.88 & 0.87 & 0.90 \\
\hline AN & 0.88 & 0.90 & 0.90 & 0.90 & 0.89 & 0.89 & 0.89 \\
\hline AG & 0.85 & 0.83 & 0.83 & 0.706 & 0.729 & 0.79 & 0.80 \\
\hline UT & 0.72 & 0.70 & 0.69 & 0.75 & 0.68 & 0.69 & 0.71 \\
\hline MO & 0.48 & 0.58 & 0.53 & 0.51 & 0.55 & 0.44 & 0.53 \\
\hline CO & 0.45 & 0.48 & 0.20 & 0.27 & 0.23 & 0.53 & 0.41 \\
\hline $\mathrm{N}$ & 147 & 309 & 197 & 142 & 181 & 174 & 1150 \\
\hline
\end{tabular}

Como criterio general George y Mallery (2003, p. 231) sugieren como recomendaciones para evaluar los coeficientes alfa de Cronbach: mayor de 0.9 es excelente, entre 0.8 y 0.9 es bueno, entre 0.7 y 0.8 es aceptable, entre 0.6 y 0.7 es cuestionable, entre 0.5 y 0.6 es pobre y menor que 0.5 es inaceptable.

- Solo los índices correspondientes al factor confianza (CO) y dos correspondientes al factor motivación (MO) resultan inaceptables, debido, quizás a que son pocos los ítems que componen cada uno de ellos.

- Los índices correspondientes a la escala de Actitud (AU) son todos excelentes o muy buenos.

\section{Medias por curso académico, por factor y total}

En la tabla 5 se presenta la media de actitud y de cada factor en el periodo analizado.

Tabla 5.

Media por curso académico

\begin{tabular}{llllllll}
\hline & $11 / 12$ & $12 / 13$ & $13 / 14$ & $14 / 15$ & $15 / 16$ & $16 / 17$ & TOT \\
\hline AU & 3.12 & 3.16 & 3.22 & 3.31 & 3.26 & 3.22 & 3.21 \\
\hline AN & 2.88 & 3.05 & 3.04 & 2.95 & 2.90 & 2.92 & 2.97 \\
\hline AG & 2.55 & 2.71 & 2.53 & 2.70 & 2.67 & 2.49 & 2.62 \\
\hline UT & 3.27 & 3.26 & 3.41 & 3.52 & 3.51 & 3.48 & 3.39 \\
\hline MO & 3.64 & 3.78 & 3.86 & 3.94 & 3.86 & 3.90 & 3.83 \\
\hline CO & 3.80 & 3.87 & 3.93 & 4.11 & 4.07 & 3.93 & 3.94 \\
\hline $\mathrm{N}$ & 147 & 309 & 197 & 142 & 181 & 174 & 1150 \\
\hline
\end{tabular}


De los valores de la tabla 4 se puede deducir que la actitud en todos los cursos académicos está por encima del valor neutral (3), entre 3.12 y 3.31, que el factor ansiedad está próximo a 3 , que el factor agrado se sitúa siempre por debajo del valor neutral, que el factor utilidad llega hasta 3.52, que los alumnos están motivados próximos al valor 4 y que los alumnos tienen confianza en las matemáticas con valores que superan el valor 4 .

\section{Medias por curso del grado, sexo y curso académico}

En la tabla 6 se presentan los resultados de actitud hacia las matemáticas por curso académico, sexo y curso del grado. El hacer la distinción por sexo es porque el $73.8 \%$ del alumnado participante en el estudio son mujeres y serán la mayoría de los futuros docentes de primaria.

Tabla 6.

Media por curso académico, sexo y curso del grado

\begin{tabular}{llllllll}
\hline AU & $11 / 12$ & $12 / 13$ & $13 / 14$ & $14 / 15$ & $15 / 16$ & $16 / 17$ & TOT \\
\hline HOM & 3.28 & 3.23 & 3.44 & 3.35 & 3.35 & 3.26 & 3.32 \\
\hline MUJ & 3.06 & 3.14 & 3.14 & 3.29 & 3.22 & 3.21 & 3.17 \\
\hline $2 .^{\circ}$ & 3.09 & 3.15 & 3.18 & 3.38 & 3.14 & 3.25 & 3.18 \\
\hline $3 .^{\circ}$ & 3.14 & 3.14 & 3.10 & 3.11 & 3.30 & 3.18 & 3.17 \\
\hline $4 .^{\circ}$ & --- & 3.21 & 3.46 & 3.36 & 3.40 & 3.27 & 3.35 \\
\hline TOT & 3.12 & 3.16 & 3.22 & 3.31 & 3.26 & 3.22 & 3.21 \\
\hline
\end{tabular}

En todos los cursos académicos la actitud hacia las matemáticas es ligeramente superior en hombres que en mujeres, variando los primeros de 3.23 a 3.44 y las segundas de 3.06 a 3.29. Por cursos académicos los estudiantes de $4 .^{\circ}$ tienen mejor actitud que sus compañeros de $2 .^{\circ}$ y $3 . .^{\circ}$ en cuatro de los seis cursos analizados.

\section{Medias por curso académico y curso del grado, según sexo}

En la tabla 7 se presentan, tanto por hombre como por mujer, las medias de actitud por curso académico y curso del grado.

Tabla 7.

Media por curso académico y curso del grado, según sexo

HOMBRE

\begin{tabular}{llllllll}
\hline AU & $11 / 12$ & $12 / 13$ & $13 / 14$ & $14 / 15$ & $15 / 16$ & $16 / 17$ & TOT \\
\hline $2 .^{\circ}$ & 3.24 & 3.16 & 3.32 & 3.55 & 3.06 & 3.19 & 3.25 \\
\hline $3 .^{\circ}$ & 3.33 & 3.40 & 3.50 & 3.00 & 3.42 & 3.13 & 3.33 \\
\hline
\end{tabular}


HOMBRE

\begin{tabular}{lccccccc}
\hline AU & $11 / 12$ & $12 / 13$ & $13 / 14$ & $14 / 15$ & $15 / 16$ & $16 / 17$ & TOT \\
\hline $4 .^{\circ}$ & -- & 3.28 & 3.54 & 3.32 & 3.46 & 3.44 & 3.43 \\
\hline \multicolumn{7}{c}{ MUJER } \\
\hline $2 .^{\circ}$ & 3.03 & 3.15 & 3.13 & 3.31 & 3.16 & 3.27 & 3.16 \\
\hline $3 .^{\circ}$ & 3.09 & 3.08 & 3.02 & 3.14 & 3.22 & 3.19 & 3.12 \\
\hline $4 .^{\circ}$ & -- & 3.20 & 3.41 & 3.38 & 3.35 & 3.13 & 3.30 \\
\hline
\end{tabular}

Los alumnos de $2 .^{\circ}$ curso, considerados por sexo, y a lo largo de los seis cursos analizados, en hombres la actitud varía de 3.06 a 3.55 y en mujeres de 3.03 a 3.31. En $3 .^{\circ}$ para hombres varía de 3 a 3.50 y en mujeres de 3.02 a 3.22. Por último, en $4 .^{\circ}$, los hombres de 3.28 a 3.54 y en mujeres de 3.13 a 3.41 , con los mejores resultados.

\section{Porcentajes actitud inferior a 3 por factor y curso académico}

Al ser el valor 3 neutral, ni de acuerdo ni en desacuerdo, se obtiene para actitud hacia las matemáticas y cada factor el porcentaje de alumnos con actitud negativa (inferior a 3). En la tabla 8 por curso académico y en la tabla 9 por sexo y curso del grado.

Tabla 8.

Porcentaje de actitud negativa (inferior a 3) por curso académico

\begin{tabular}{lllllll}
\hline & $11 / 12$ & $12 / 13$ & $13 / 14$ & $14 / 15$ & $15 / 16$ & $16 / 17$ \\
\hline AU & 43.54 & 39.81 & 32.49 & 25.35 & 28.18 & 32.18 \\
\hline AN & 53.06 & 44.98 & 46.70 & 48.59 & 53.59 & 55.17 \\
\hline AG & 63.95 & 57.28 & 62.44 & 57.75 & 61.33 & 70.11 \\
\hline UT & 27.89 & 28.80 & 21.83 & 16.20 & 15.47 & 14.94 \\
\hline MO & 12.24 & 9.06 & 6.09 & 4.23 & 5.52 & 5.17 \\
\hline CO & 9.52 & 7.77 & 3.05 & 1.41 & 2.76 & 6.32 \\
\hline A20 & 8.16 & 9.39 & 5.58 & 1.41 & 3.31 & 6.90 \\
\hline A9 & 61.90 & 58.90 & 58.88 & 51.41 & 52.49 & 58.05
\end{tabular}

- El porcentaje de alumnos con puntuación inferior a 3 en actitud hacia las matemáticas (AU) varía del $25.35 \%$ al $43.54 \%$.

- $\quad$ En el factor ansiedad (AN) va de $44.98 \%$ a $55.17 \%$. 
- En el factor agrado (AG) va del $57.28 \%$ al $70.11 \%$.

- En el factor utilidad (UT) va del $14.94 \%$ al $28.80 \%$.

- En el factor motivación (MO) va del $4.23 \% 12.24 \%$.

- En el factor confianza (CO) va del $1.41 \%$ al $9.52 \%$.

- En el I ítem mejor puntuado A2o la puntuación inferior a 3 va del $2.41 \%$ al 9.39\%. Y en el peor valorado $A 9$ va del $51.41 \%$ al $61.90 \%$.

Uno de cada cuatro alumnos en el curso mejor (14/15) y dos de cada cinco en el curso peor (11/12), tienen una actitud negativa hacia las matemáticas.

Tabla 9.

Porcentaje de actitud negativa (inferior a 3) por sexo y curso del grado

\begin{tabular}{lcccccc}
\hline & HOM & MUJ & $2 .^{\circ}$ & $3 .^{\circ}$ & $4 .^{\circ}$ & TOT \\
\hline AU & 26.25 & 37.10 & 35.04 & 38.03 & 25.00 & 34.26 \\
\hline AN & 48.50 & 50.06 & 48.03 & 52.11 & 48.61 & 49.65 \\
\hline AG & 55.81 & 63.72 & 60.04 & 65.49 & 57.87 & 61.65 \\
\hline UT & 27.89 & 28.80 & 21.83 & 16.20 & 15.47 & 21.74 \\
\hline MO & 7.64 & 7.07 & 7.09 & 7.75 & 6.48 & 7.22 \\
\hline CO & 2.99 & 6.12 & 6.10 & 5.63 & 2.78 & 5.30 \\
\hline A20 & 5.31 & 6.60 & 7.09 & 6.10 & 4.63 & 6.26 \\
\hline A9 & 50.17 & 59.72 & 58.46 & 59.62 & 49.54 & 57.22 \\
\hline
\end{tabular}

- Es en $4 .^{\circ}$ curso en donde los porcentajes de puntuación inferior a 3 (actitud negativa), son inferiores que en $2 .^{\circ}$ y $3 .^{\circ}$, en todas las variables estudiadas.

- Por sexo los alumnos tienen porcentajes más bajos que las alumnas en todas las variables, menos en motivación.

- Uno de cada tres futuros maestros tiene una actitud negativa hacia las matemáticas.

- A uno de cada dos alumnos del GMP no le divierte hablar con otros de matemáticas.

\section{Diferencias por sexo, curso del grado y curso académico}

Hay diferencias significativas $(p<.001)$ en actitud hacia las matemáticas a favor de hombres (3.32) frente a mujeres (3.17). También en agrado a favor de hombres (2.74) frente a mujeres (2.58). En el ítem peor valorado se encuentran diferencias significativas, pues a los hombres (2.42) les divierte hablar con otros de matemáticas más que a las mujeres (2.19).

Y en motivación las mujeres (3.86) están más motivadas que los hombres (3.72). Por el contrario no se encuentran diferencias significativas en ansiedad $(p=.243)$, ni en utilidad $(p=.482)$, ni en confianza ( $p=.243)$, ni tampoco en el ítem A20 ( $p=.517)$. 
Por Curso del Grado hay diferencias significativas favorables a $4 .^{\circ}$ en actitud, en utilidad y en el ítem A9. Los alumnos de $3 .^{\circ}$ tienen en agrado y motivación una diferencia significativa desfavorable, mientras que en ansiedad $(p=.407)$, confianza $(p=.431)$ y A20 $(p=.407)$ no hay diferencias significativas.

Por Curso Académico, resulta que en actitud hay diferencia significativa favorable en el curso $14 / 15(p=.018)$, en ansiedad no hay diferencias significativas $(p=.101)$, en agrado hay diferencias favorable en los curso 12/13, 14/15 y 15/16 ( $p=.008)$, en el factor utilidad favorable en los curso 14/15, 15/16 y 16/17 ( $p<.001$ ), en motivación favorable en el curso 14/15 ( $p=.001)$ y en confianza favorable en el curso 14/15 ( $p<.001)$. En A20 es desfavorable en 12/13 ( $p=.001)$ y en $A 9$ no hay diferencias significativas. Actitud y utilidad favorable a los alumnos de $4 .^{\circ}$ curso. Y actitud, motivación y confianza mejor en el curso 14/15.

\section{Correlación entre factores y regresión múltiple}

Se efectúa la correlación de los cinco factores entre ellos y con actitud, obteniendo como resultados:

- agrado con utilidad (.560) y con motivación (.305) correlaciones moderada y baja, respectivamente.

- utilidad con confianza (.498) y con motivación (.509) moderadas.

- actitud con confianza (.532) y con motivación (.582) moderadas.

- confianza con agrado (.425) moderada y con motivación (.344) baja.

- actitud con agrado (.769) y con utilidad (.738) correlaciones altas.

Calculada la correlación entre edad y actitud resulta un valor de $r=.155$, que es una correlación muy baja, aunque significativa $(p<.001)$. No hay una relación entre edad y actitud que pueda indicarnos si los alumnos más jóvenes tienen mayor actitud hacia las matemáticas o viceversa.

Posteriormente se obtiene una regresión múltiple para conocer en qué medida influye cada uno de los cinco factores en la actitud hacia las matemáticas, encontrando:

$$
A U=0.243+0.039 * A N+0.330 * A G+0.257 * U T+0.145 * M O+0.150 * C O \quad(R=.884)
$$

En donde todas las variables independientes son significativos y los dos factores que mayor peso tienen son agrado y utilidad, cuyas correlaciones con actitud son las más altas.

\section{Discusión y Conclusiones}

Siguiendo la clasificación de Sánchez, Segovia y Miñán (2011) que establecen tres categorías, nivel medio las puntuaciones comprendidas en el intervalo (Media-DT, Media+DT), nivel bajo las puntuaciones inferiores a este intervalo y nivel alto las superiores, en el intervalo medio se encuentra el $68.52 \%$, en el inferior el $15.57 \%$ del total y en el superior el $15.91 \%$. De ahí que el $84.09 \%$ de los alumnos del GMP no tengan una actitud media-alta hacia las matemáticas.

El ítem mejor valorado es A20 con 4.33 y el peor valorado es A9 con 2.25. No ratifican estos resultados los de Flores y Auzmendi (2015) que obtuvieron como ítem más valorado A1 (Considero las matemáticas como una materia muy necesaria en mis estudios) con 4.200 y el menos A4 (Utilizar las matemáticas es una diversión para mí) con 2.89o. Sin embargo, Somohano (2015) obtuvo que el ítem "Cuando resuelvo un problema difícil de Matemáticas siento satisfacción personal" que se corresponde con el A20 (Me provoca una gran satisfacción el llegar a resolver problemas de matemáticas) es el más valorado con 4.22, 
algo inferior al obtenido en este estudio. En el otro extremo Maz-Machado et al. (2014) coincide con el presente estudio al considerar menos valorado el ítem A9 (Me divierte hablar con otros de matemáticas) con 2.35.

Por factores agrado (2.62), utilidad (3.39) y motivación (3.83) están en la misma línea que Maz-Machado et al. (2014) que son respectivamente 2.66, 3.38 y 3.68, un poco alejados de Flores y Auzmendi (2015) con 3.112, 3.479 y 3.363, respectivamente.

Por fiabilidad en todos los cursos se sitúa entre 0.87 y 0.92 , lo que indica que es buena 0 excelente, entre los que se encuentra el 0.914 de Flores y Auzmendi (2015) y el de Casas et al. (2016) de 0.887 e inferiores al de Auzmendi (1992) de 0.928 y próximo al de LeónMantero et al. (2015) de 0.919. Por tanto, la fiabilidad de la escala es buena en todo el periodo.

Considerando las tres variables de corte, por sexo hay diferencias significativas en actitud y agrado favorable a hombres, en motivación a favor de mujeres y en ansiedad, utilidad y confianza no hay diferencias significativas. Por curso del grado hay diferencias significativas favorables a los alumnos de $4 .^{\circ}$ en actitud y utilidad, desfavorable para $3 .^{\circ}$ en agrado y motivación y no hay diferencias significativas en ansiedad y confianza. $Y$ por curso académico, el 2014/15 es favorable en actitud, agrado, utilidad, motivación y confianza.

Las correlaciones entre factores son más fuerte en Flores y Auzmendi (2015) y Casas et al. (2016) que en el presente estudio, quizás debido a que las muestras utilizadas en los casos de referencia son más pequeñas y en una sola toma de datos, mientras que aquí es a lo largo de seis cursos académicos; sin embargo, en los tres casos las correlaciones obtenidas son significativas. También se ha analizado si la actitud hacia las matemáticas está relacionada con la edad de los futuros maestro siendo una correlación muy baja, aunque significativa, resultado cercano al obtenido por Estrada y Diez-Palomar (2011) que indican que "sentimiento hacia las matemáticas y edad son dos aspectos independientes" (p. 130). Agrado-confianza, una vez estructurados los ítems, es la correlación más alta entre factores (.747) en León-Mantero et al. (2018) y la más baja motivación-confianza (.198), mientras que los obtenidos en el presente estudio con los ítems originales del cuestionario es de $.425 \mathrm{y}$ .344, respectivamente. Es importante resaltar que los factores que más influyen en el resultado de la actitud son agrado y utilidad, según la recta de regresión múltiple obtenida, siendo la correlación entre ambos más alta entre factores.

Este estudio no reafirma lo dicho por Blanco (2015) en cuanto a que "en general, los futuros profesores de primaria muestran una actitud negativa hacia las matemáticas" (p. 11), ya que uno de cada tres alumnos tiene una puntuación inferior a 3 y en los seis cursos académicos analizados la actitud media sufre muy pocas variaciones (va de 3.12 a 3.31) inferiores al 3.373 de Flores y Auzmendi (2015) y superiores al 3.014 de Fernández y Aguirre (2010), por lo que parece ser algo estable entre los estudiantes que acceden al GMP.

Los participantes de este estudio tienen una ansiedad neutra, a dos de cada tres alumnos les agradan poco las matemáticas, uno de cada cinco no le encuentra utilidad a las matemáticas, uno de cada diez no se siente motivado y uno de cada veinte no tiene confianza en las matemáticas. Además a los alumnos a los que les agradan las matemáticas tienen una correlación alta con actitud ( $\mathrm{r}=.769)$ y también los que las consideran útiles con actitud ( $r=.738)$, y que esta situación de más utilidad que agrado "se viene repitiendo año tras año desde la implantación del Grado de Maestro de Primaria" (AAA y BBB, 20**, p. 375) por lo que habría que aumentar el número de alumnos a los que agraden las matemáticas para que de esta forma aumentara su actitud hacia las matemáticas y quizás una selección previa del alumnado con una prueba de aptitud ayudaría a la formación inicial de maestros que realizarán sus estudios sin un rechazo a las Matemáticas. 
Queda abierto el presente estudio a analizar la reordenación y eliminación de ítems siguiendo propuestas de otros autores por si fuera necesario ajustar los resultados a otras clasificaciones en factores.

\section{Bibliografía}

$\mathrm{AAA}$ y $\mathrm{BBB}\left(20^{* *}\right)$

Alemany, I. y Lara, A. I. (2010). Las actitudes hacia las matemáticas en el alumnado de ESO: un instrumento para su medición. Publicaciones, 40, 49-70.

Auzmendi, E. (1992). Las actitudes hacia la Matemática-Estadística en las Enseñanzas Medias y Universitaria. Bilbao: Mensajero.

Blanco, L. (2015). Resolución de problemas de matemáticas. En L. Blanco, J. Cárdenas y A. Caballero (Coord.), La resolución de problemas de matemáticas en la formación inicial de profesores de primaria, 11-22. Manuales UNEX, n. ${ }^{\circ}$ 98, Badajoz: Servicio de Publicaciones Universidad de Extremadura.

Casas, J. C., León-Mantero, C., Maz-Machado, A., Jiménez-Fanjul, N. y Madrid M. J. (2016). Identificando las relaciones de la escala de actitud hacia las matemáticas propuesta por Auzmendi en maestros en formación. En J. A. Macías, A. Jiménez, J. L. González, M. T. Sánchez, P. Hernández, C. Fernández, F. J. Ruiz, T. Fernández y A. Berciano (Eds.), Investigación en Educación Matemática XX (p. 579). Málaga: SEIEM.

Estrada, A. y Diez-Palomar, J. (2011). Las actitudes hacia las Matemáticas. Análisis descriptivo de un estudio de caso exploratorio centrado en la educación matemática de familiares. Revista de Investigación en Educación, 9(2), 116-132.

Fennema, E. y Sherman, J. (1976). Fennema-Sherman Mathematics Attitudes: Instruments Designed to Measure Attitudes Toward the Learning of Mathematics by Males and Females. JSAS Catalog of Selected Documents in Psychology, 6, 31 (Ms. No. 1255). Journal for Research in Mathematics Education, 7, p. 324-326.

Fernández, R. y Aguirre, C. (2010). Actitudes iniciales hacia las matemáticas en alumnos de grado de Magisterio de Educación Primaria: Estudio de una situación en el EEES. Unión, 23, 107-116.

Fernández Cézar, R., Solano Pinto, N., Rizzo, K., Gomezescobar, A., Iglesias, L. M. y Espinosa, A. (2016). CTS, 33(11), 227-238

Flores, W. O. y Auzmendi, E. (2015). Análisis de la estructura factorial de una escala de actitud hacia las matemáticas. Aula de encuentro, 17(1), 45-77.

George, D. y Mallery, P. (2003). Spss for Windows step by step: A Simple Guide and Reference. 11.0 Update ( $4 .^{\mathrm{a}}$ ed.). Boston: Allyn \& Bacon.

Gómezescobar Camino, A. y Fernández Cézar, R. (2018). Los maestros y sus actitudes hacia las Matemáticas: un estudio sobre Educación Infantil y Primaria en España. Unión, $52,186-200$ 
León-Mantero, C., Pedrosa-Jesús, C., Maz-Machado, A. y Casas-Rosal, J. C. (2018). Tratamiento matemático de mediciones de actitudes con escalas tipo Likert. En L. J. Rodríguez-Muñiz, L. Muñiz-Rodriguez, A. Aguilar-González, P. Alonso, F. J. García García y A. Bruno (Eds.), Investigación en Educación Matemática XXII (pp. 290-299). Gijón: SEIEM.

León-Mantero, C., Maz-Machado, A. y Jiménez-Fanjul, N. (2015). Identificando las actitudes hacia las matemáticas en los estudiantes para maestros. JAEM 17. Cartagena (Murcia).

Martínez, O. J. (2008). Actitudes hacia la matemática. Revista Universitaria de Investigación $9(1), 237-256$.

Maz-Machado, A., León-Mantero, C., Casas, J. C. y Gática, S. N. (2014). Actitudes hacia las matemáticas: un estudio en alumnos de Ingeniería Informática. XV Congreso de Enseñanza y Aprendizaje de las Matemáticas. Baeza (Jaén).

Muñoz, J. M. y Mato, M. D. (2008). Análisis de las actitudes respecto a las matemáticas en alumnos de ESO. Revista de Investigación Educativa, 26(1), 209-226.

Palacios, A., Arias, V. y Arias, B. (2014). Las actitudes hacia las matemáticas: construcción y validación de un instrumento para su medida. Revista de Psicodidáctica, 19(1), 67-91.

Real Decreto 126/2014, de 28 de febrero, por el que se establece el currículo básico de la Educación Primaria. Boletín Oficial del Estado. Madrid, 1 de marzote 2014, núm. 52, pp. 19349-19420

Sánchez, J., Segovia, I. y Miñán, A. (2011). Exploración de la ansiedad hacia las matemáticas en los futuros maestros de educación primaria. Revista Profesorado 15(3), 297-312. 


\section{Anexo}

Cuestionario de Actitud hacia las Matemáticas de Auzmendi (1992)

A1 Considero las matemáticas como una materia muy necesaria en mis estudios.

A2 La asignatura de matemáticas se me da muy mal.

A3 Estudiar o trabajar con las matemáticas no me asusta en absoluto.

A4 Utilizar las matemáticas es una diversión para mí.

A5 La matemática es demasiado teórica para que pueda servirme de algo.

A6 Quiero llegar a tener un conocimiento más profundo de las matemáticas.

A7 Las matemáticas es una de las asignaturas que más temo.

A8 Tengo confianza en mí cuando me enfrento a un problema de matemáticas.

A9 Me divierte el hablar con otros de matemáticas.

A10 Las matemáticas pueden ser útiles para el que decida realizar una carrera de "ciencias", pero no para el resto de los estudiantes.

A11 Tener buenos conocimientos de matemáticas incrementará mis posibilidades de trabajo.

A12 Cuando me enfrento a un problema de matemáticas me siento incapaz de pensar con claridad.

A13 Estoy calmado/a y tranquilo/a cuando me enfrento a un problema de matemáticas.

A14 Las matemáticas son agradables y estimulantes para mí.

A15 Espero tener que utilizar poco las matemáticas en mi vida profesional.

A16 Considero que existen otras asignaturas más importantes que las matemáticas en mi futura profesión.

A17 Trabajar con las matemáticas hace que me sienta muy nervioso/a.

A18 No me altero cuando tengo que trabajar en problemas de matemáticas.

A19 Me gustaría tener una ocupación en la cual tuviera que utilizar las matemáticas.

A20 Me provoca una gran satisfacción el llegar a resolver problemas de matemáticas.

A21 Para mi futuro profesional la matemática es una de las asignaturas más importantes que tengo que estudiar.

A22 Las matemáticas hacen que me sienta incómodo/a y nervioso/a.

A23 Si me lo propusiera creo que llegaría a dominar bien las matemáticas.

A24 Si tuviera oportunidad me inscribiría en más cursos de matemáticas de los que son obligatorios.

A25 La materia que se imparte en las clases de matemáticas es muy poco interesante. 